\title{
Resultados de un programa de control de calidad externo del tamizaje serológico de anticuerpos contra Trypanosoma cruzi en donantes de sangre de Brasil
}

\author{
Amadeo Sáez-Alquézar, ${ }^{1}$ Márcia Murta, ${ }^{1}$ Waldelania Pereira Marques ${ }^{1}$ \\ y Guilherme Rodrigues da Silva ${ }^{2}$
}

RESUMEN Objetivos. A partir de 1995 en Brasil se iniciaron programas de control de calidad externo de laboratorios de serología (PCCES) abiertos a la participación de laboratorios de bancos de sangre públicos y privados. Estos programas se han puesto en práctica tres veces al año desde 1995 y cuentan con la participación de más de 100 entidades. El objetivo de este trabajo es analizar los resultados de los ocho últimos programas de evaluación del tamizaje serológico para la enfermedad de Chagas, que se realizaron entre abril de 1999 y agosto de 2001.

Métodos. Participaron en los ocho programas laboratorios de serología de instituciones públicas y privadas. El número de laboratorios participantes que entregó los resultados en cada uno de los ocho PCCES fue de 94, 90, 85, 94, 100, 103, 102 y 116, respectivamente. Al inicio de cada PCCES se envió un panel enmascarado a cada institución participante, con un plazo de 60 dias para informar los resultados del procesamiento de las muestras. Se utilizaron pruebas de inmunoadsorción enzimática (ELISA), inmunofluorescencia indirecta (IFI) y hemaglutinación indirecta (HAI). Posteriormente, cada institución recibió del centro organizador (PANEL) la clave con los resultados correctos para su autoevaluación. Los paneles estaban compuestos por 24 muestras de sueros con diferentes reactividades a los marcadores obligatorios en el tamizaje serológico de donantes de sangre en Brasil, incluido el de muestras negativas.

Resultados. La técnica de ELISA fue la más utilizada en el tamizaje (92\%-98\%). La estrategia de tamizaje más usada por los laboratorios participantes fue la combinación de una prueba de ELISA y una de HAI (58\%-83\%). La mayoría de los laboratorios participantes obtuvieron resultados correctos en los diferentes programas sin resultados negativos falsos (83,6\%-98,1\%). De las 5406 muestras de suero positivas a anti-Trypanosoma cruzi que hubo en los ocho programas, 85 (1,6\%) fueron notificadas de negativas por 58 laboratorios, pero solamente 14 de ellos dejaron de identificar una o más muestras positivas por no conseguir detectarlas por medio de ninguna de las pruebas de tamizaje utilizadas. No se observó ninguna diferencia significativa entre bancos en los ocho programas $(\mathrm{P}=0,5936)$. No se observó asociación entre los resultados negativos falsos y una muestra o sistema diagnóstico en particular. La técnica de HAI presentó el mayor número de resultados negativos falsos $(0,7 \%-7,9 \%)$. De los 32855 resultados obtenidos en muestras de sueros negativas a antiT. cruzi en los ocho programas, $106(0,32 \%)$ fueron notificados como resultados positivos

1 Panel Control de Calidad S/C Ltda., São Paulo, Brasil. La correspondencia debe dirigirse a Amadeo Sáez-Alquézar a la siguiente dirección postal: Panel Controle de Qualidade S/C Ltda., Rua
Dr. Luís Migliano No. 2050, Morumbi, São Paulo, SP, Brasil, CEP:05711-001. Correo electrónico: amadeosaez@uol.com.br
2 Facultad de Medicina, Universidad de São Paulo, SP, Brasil. 
Palabras clave falsos. Entre $1 \%$ y $16 \%$ de los laboratorios participantes obtuvieron un resultado positivo falso por programa y entre $0 \%$ y $4 \%$ notificó más de un resultado positivo falso. La proporción de resultados negativos falsos muestra una tendencia descendente después de 1995.

Conclusiones. Los resultados obtenidos en este trabajo apuntan hacia una tendencia desecendente en la proporción de resultados negativos falsos. Al mismo tiempo se observó que la técnica de HAI continúa arrojando el mayor número de resultados negativos falsos. Se sugiere darle preferencia a la técnica ELISA para el tamizaje de donantes de sangre.

Tamizaje serológico, bancos de sangre, control de calidad, serología, programas de evaluación, anticuerpos anti-Trypanosoma cruzi, enfermedad de Chagas.
Durante el período de 1994 a 1995, el Ministerio de Salud de Brasil desarrolló cuatro programas de control de calidad externo en serología (PCCES) para bancos de sangre públicos. Los resultados observados en esos programas en relación con el tamizaje serológico de anti-Trypanosoma cruzi mostró una mejora importante (1). Aun así, en aquella ocasión se observaron 64 $(3,7 \%)$ resultados negativos falsos, siendo mayor su incidencia (49) con las pruebas de hemoaglutinación indirecta (HAI).

A partir de 1995, PANEL Control de Calidad $^{3}$ (PANEL), junto con la Sociedad Brasileña de Hematología y Hemoterapia (SBHH), inició PCCES abiertos a la participación de laboratorios de serología de bancos de sangre públicos y privados de Brasil. Estos programas se realizan tres veces al año desde 1995, y actualmente cuentan con la participación de más de cien laboratorios.

El objetivo de este trabajo es analizar los resultados de los ocho últimos programas para la evaluación del tamizaje serológico para la enfermedad de Chagas, realizados entre abril de 1999 y agosto de 2001.

\section{MATERIALES Y MÉTODOS}

\section{Participantes de los PCCES}

En el presente trabajo se consideran los ocho PCCES realizados entre

PANEL Control de Calidad es una empresa privada de Brasil que desarrolla procedimientos de control de calidad para laboratorios de serología (http://www.panel.com.br). abril de 1999 y agosto de 2001, codificados como PA0499, PA0899, PA0200, PA0400, PA0800, PA1200, PA0401 y PA0801 (las letras iniciales identifican el centro organizador del programa PANEL, los dos primeros dígitos corresponden al mes en que se realizó el programa y los dos últimos dígitos, al año).

En la figura 1 se presenta el número de laboratorios de serología, públicos y privados, a los que se enviaron muestras en cada uno de los PCCES, así como el porcentaje de ellos que informaron sus resultados, mientras que en el cuadro 1 se presentan las características de estos últimos.

\section{Desarrollo de los programas}

El centro organizador envió un panel enmascarado a cada institución participante para que fuera procesado tal como se tamizan las muestras de donantes de sangre. Junto con el panel se enviaron instrucciones detalladas para el procesamiento de las muestras.

Para la devolución de los resultados se facilitaron cuadernos con espacios adecuados y disquetes formateados, de modo que los resultados pudieran enviarse por correo normal o electrónico en un plazo de 60 días después del procesamiento de las muestras del panel.

FIGURA 1. Número de laboratorios participantes y porcentajes de laboratorios que notificaron resultados en los programas de control de calidad externos de laboratorios de serología, Brasil, 1999-2001

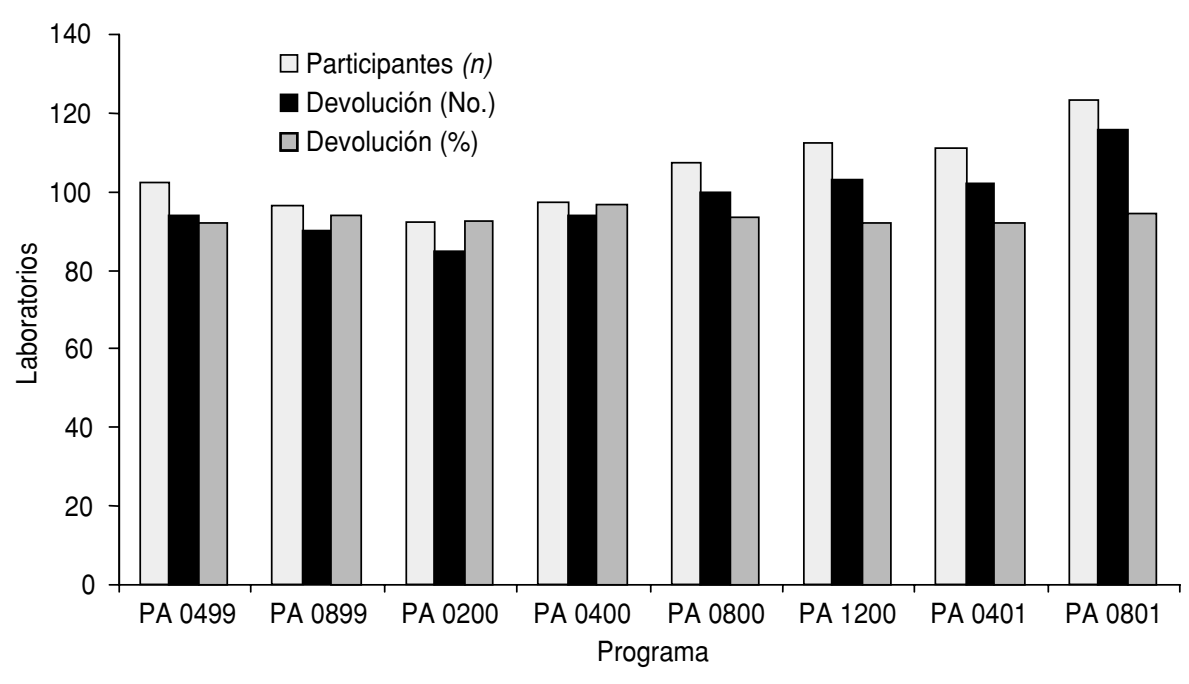


CUADRO 1. Características de los laboratorios que participaron en los programas de control de calidad externo de laboratorios de serología y que notificaron sus resultados. Brasil, 1999-2001

\begin{tabular}{|c|c|c|c|c|c|c|c|c|c|c|c|c|c|c|c|c|}
\hline \multirow[b]{3}{*}{ Tipo de laboratorio } & \multicolumn{16}{|c|}{ Programa } \\
\hline & \multicolumn{2}{|c|}{ PA0499 } & \multicolumn{2}{|c|}{ PA0899 } & \multicolumn{2}{|c|}{ PA0200 } & \multicolumn{2}{|c|}{ PA0400 } & \multicolumn{2}{|c|}{ PA0800 } & \multicolumn{2}{|c|}{ PA1200 } & \multicolumn{2}{|c|}{ PA0401 } & \multicolumn{2}{|c|}{ PA0801 } \\
\hline & No. & $\%$ & No. & $\%$ & No. & $\%$ & No. & $\%$ & No. & $\%$ & No. & $\%$ & No. & $\%$ & No. & $\%$ \\
\hline Públicos (BS) & 35 & 37,2 & 32 & 35,6 & 28 & 33 & 30 & 31,9 & 40 & 40 & 38 & 36,9 & 39 & 38,2 & 45 & 38,8 \\
\hline Privados ( $\left(\mathrm{CC}^{\mathrm{b}}\right)$ & 17 & 18,2 & 16 & 17,8 & 17 & 20 & 17 & 18,2 & 17 & 17 & 18 & 17,5 & 18 & 17,6 & 19 & 16,4 \\
\hline Públicos (LC) & 4 & 4,2 & 2 & 2,2 & 3 & 3,5 & 3 & 3,2 & 2 & 2 & 3 & 2,9 & 4 & 3,9 & 4 & 3,4 \\
\hline
\end{tabular}

a BS: bancos de sangre.

b LC: laboratorio clínico.

Después de informar los resultados, cada institución recibió del centro organizador la clave con los resultados correctos para su autoevaluación.

Una vez recibidos los resultados de los laboratorios participantes, el centro organizador los analizó y preparó un informe final que fue enviado a todos los participantes. En él se hicieron constar: a) las características del panel utilizado con la clave y con las metodologías usadas en la caracterización; b) las estrategias, metodologías y marcas comerciales utilizadas por los participantes; c) el número de muestras negativas y positivas, y la cantidad y el porcentaje de resultados negativos $y$ positivos falsos; d) la distribución de los resultados negativos y positivos falsos según la técnica empleada y marca comercial.

Durante el desarrollo de los PCCES se mantuvo el más estricto grado de confidencialidad con respecto a los resultados individuales obtenidos por cada institución participante.

\section{Paneles}

Los paneles utilizados contenían 24 muestras de sueros con diversas reactividades a los marcadores de uso obligatorio en el tamizaje serológico de donantes de sangre en Brasil (1), incluidas las muestras negativas. En el cuadro 2 se presenta la composición de los paneles utilizados en los ocho PCCES.

La confección de los paneles de sueros fue realizada en PANEL a partir de unidades de plasma que fueron descartadas en diversos bancos de sangre por haber presentado algún tipo de reactividad en el tamizaje serológico de los donantes. A la confección de los paneles le siguió el procedimiento ya descrito $(2,3)$, que abarca diversas etapas: recalcificación, centrifugación, diálisis, centrifugación y filtrado. El conservante utilizado en las muestras del panel fue Bronidox-L. Los paneles se distribuyeron en estuches térmicos con hielo reciclable en su interior.

Caracterización de los paneles. El número de muestras positivas a antiT. cruzi en cada panel fue:

PA0499: 4

PA0899: 3 y 4 (se utilizaron

PA0200: 3

2 paneles distintos)

PA0400: 3

PA0800: 4

PA1200: 3

PA0401: 2

PA0801: 2

Cada una de las muestras de suero del panel fue analizada mediante pruebas serológicas para detectar los marcadores de uso obligatorio en bancos de sangre, usando pruebas de diferentes casas comerciales, incluidas las confirmatorias, a manera de garantizar la mayor confiabilidad en la clasificación de cada muestra como positiva o negativa a cada marcador. La caracterización de las muestras positivas a anti-T. cruzi fue realizada al menos mediante dos pruebas de ELISA, dos de HAI y una de inmunofluorescencia indirecta (IFI).

Todas las muestras positivas evaluadas por ELISA ofrecieron una relación de densidad óptica (DO)/Valor de Corte $(\mathrm{VC})>2$; para las pruebas de HAI e IFI, los resultados positivos presentaron títulos superiores a 1/20. El resto de las muestras del panel fueron negativas a anti-T. cruzi (ELISA: $\mathrm{DO} / \mathrm{VC}<0,8$; HAI y IFI: resultados negativos con título inferior a $1 / 20$ ).

\section{Criterio de evaluación}

El criterio de evaluación utilizado por marcador e institución tomó en consideración la presencia de resultados positivos y negativos falsos, y se emplearon las siguientes categorías:

A: Todos los resultados correctos, sin la presencia de resultados positivos o negativos falsos

B: Presencia de resultados positivos falsos solamente 
CUADRO 2. Composición de los paneles (reactividad de las muestras) utilizados en los programas de control de calidad externo de laboratorios de serología. Brasil, 1999-2001

\begin{tabular}{|c|c|c|c|c|c|c|c|c|c|}
\hline \multirow[b]{2}{*}{ Muestra } & \multicolumn{9}{|c|}{ Programa } \\
\hline & PA0499 & $\begin{array}{c}\text { PA0899 } \\
\text { (panel azul) }\end{array}$ & $\begin{array}{c}\text { PA0899 } \\
\text { (panel rojo) }\end{array}$ & PA0200 & PA0400 & PA0800 & PA1200 & PA0401 & PA0801 \\
\hline 2 & $C+D$ & E & $A+B$ & $E$ & D & $A$ & $C+D$ & $\mathrm{~F}$ & $A+B$ \\
\hline 3 & $C+D$ & B & $G$ & $\mathrm{~N}$ & E & A & $\mathrm{N}$ & $C+D$ & $D+F$ \\
\hline 4 & $C+D$ & A & $C+D$ & $\mathrm{~F}$ & G & $E+D$ & $\mathrm{E}$ & $\mathrm{N}$ & G \\
\hline 5 & $\mathrm{~N}$ & $C+D$ & D & $D+G$ & A & $C+D$ & $A+B+D+G$ & $C+D$ & $\mathrm{E}$ \\
\hline 8 & E & $\mathrm{G}^{\mathrm{a}}$ & $\mathrm{F}$ & A & $\mathrm{N}$ & $\mathrm{F}$ & A & $B+C$ & $D+E$ \\
\hline 9 & $\mathrm{E}$ & $\mathrm{G}^{\mathrm{a}}+\mathrm{E}$ & B & E & $E$ & A & $A+E$ & E & A \\
\hline 10 & E & $A+B+E+C+D$ & $C+D$ & $A+B$ & $\mathrm{~F}$ & $B+D+E$ & $\mathrm{~N}$ & G & $C+D$ \\
\hline 11 & G & $\mathrm{F}$ & $\mathrm{N}$ & $C+D$ & D & $\mathrm{E}$ & $\mathrm{F}$ & G & A \\
\hline 12 & G & $C+D$ & $D+G$ & $\mathrm{~F}$ & $C+D+E$ & $\mathrm{~F}$ & $\mathrm{~F}$ & A & $B+D$ \\
\hline 13 & A & $\mathrm{N}$ & $\mathrm{F}$ & $\mathrm{N}$ & A & $\mathrm{F}$ & $\mathrm{E}$ & $A+D$ & $\mathrm{E}$ \\
\hline 18 & B & $C+D$ & $\mathrm{~N}$ & D & $C+D$ & $\mathrm{~N}$ & $B+G$ & $D+G$ & $E+F$ \\
\hline 19 & B & B & D & E & A & B & $G$ & D & $A+D$ \\
\hline 20 & B & $\mathrm{E}$ & $\mathrm{F}$ & A & E & A & $D+E$ & $\mathrm{~N}$ & E \\
\hline 21 & $\mathrm{~F}$ & D & E & D & D & E & $A$ & B & B \\
\hline 22 & $\mathrm{~F}$ & A & $A+D+E$ & A & $D+F$ & $A+\bar{D}+E$ & $B+C+D$ & A & A \\
\hline 23 & $\mathrm{~F}$ & E & D & E & $C+D$ & $C+D$ & $E$ & E & $C+D$ \\
\hline 24 & $\mathrm{~F}$ & $C+D$ & $C+D$ & $C+D$ & $D+E$ & $\mathrm{~F}$ & $C+D$ & $\mathrm{E}$ & $\mathrm{N}$ \\
\hline
\end{tabular}

Nota: N: Negativo; A: anti-VIH +; B: anti-HTLV +; C: HBsAg +; D: anti-HBC +; E: anti-HCV +; F: anti-Trypanosoma cruzi +; G: sífilis +.

a Solamente para pruebas para treponema.

C: Presencia de resultados negativos falsos solamente.

\section{Análisis estadístico}

La prueba exacta de Fisher se aplicó mediante StatXact 4 (1). El análisis de las proporciones se realizó mediante el programa Pack Stata 7.0 (2). En el análisis de tendencia linear se utilizó el programa Ptrend (3).

\section{RESULTADOS}

\section{Estrategias de tamizaje}

En la figura 1 se presentan el número y el porcentaje de participantes que informaron los resultados dentro del plazo establecido en cada programa. En general, hubo una buena respuesta (entre $91,9 \%$ y $96,9 \%$ ).
Las pruebas de ELISA fueron las más utilizadas en el tamizaje (92-98\%) y la estrategia más utilizada para antiT. cruzi fue una prueba de inmunoadsorción enzimática (ELISA) más una prueba de hemaglutinación indirecta (HAI) $(58 \%-83 \%)$. La técnica de IFI asociada con otras pruebas se usó menos $(18 \%-34 \%)$ (figura 2$)$.

\section{Evaluación de los laboratorios participantes}

En la figura 3 se observa el porcentaje de laboratorios participantes, por programa, con evaluaciones $\mathrm{A}, \mathrm{B}$ y C para el tamizaje de anti-T. cruzi.

Resultados negativos falsos. Más de $80 \%$ de los laboratorios participantes obtuvieron resultados correctos $(91,5 \%$; $95,6 \%$; 97,6\%; 93,6\%; 93\%; 98,1\%;
$90,2 \%$ y $83,6 \%$ respectivamente por programa) sin la presencia de resultados negativos falsos en el procesamiento de las muestras de sueros positivas a anti-T. cruzi.

De las 5406 determinaciones realizadas en muestras de sueros positivas a anti-T. cruzi durante los 8 programas, $85(1,6 \%)$ fueron notificadas de negativas. Estos 85 resultados negativos falsos fueron obtenidos en 58 laboratorios, pero solamente 14 de ellos no pudieron identificar una o más muestras positivas mediante ninguna de las pruebas de tamizaje utilizadas (cuadro 3). Por lo tanto, el porcentaje de laboratorios con resultados negativos falsos fue muy bajo $(0 \%-3,4 \%)$ y no se observaron diferencias significativas entre el comportamiento de los laboratorios participantes $(P=0,5936)$.

En el cuadro 3 se puede observar el número total de resultados negativos falsos obtenidos por los laboratorios 
FIGURA 2. Pruebas o combinaciones de pruebas serológicas para el tamizaje de antiTrypanosoma cruzi utilizadas por los laboratorios que participaron en los programas de control de calidad externos de laboratorios de serología, Brasil, 1999-2001

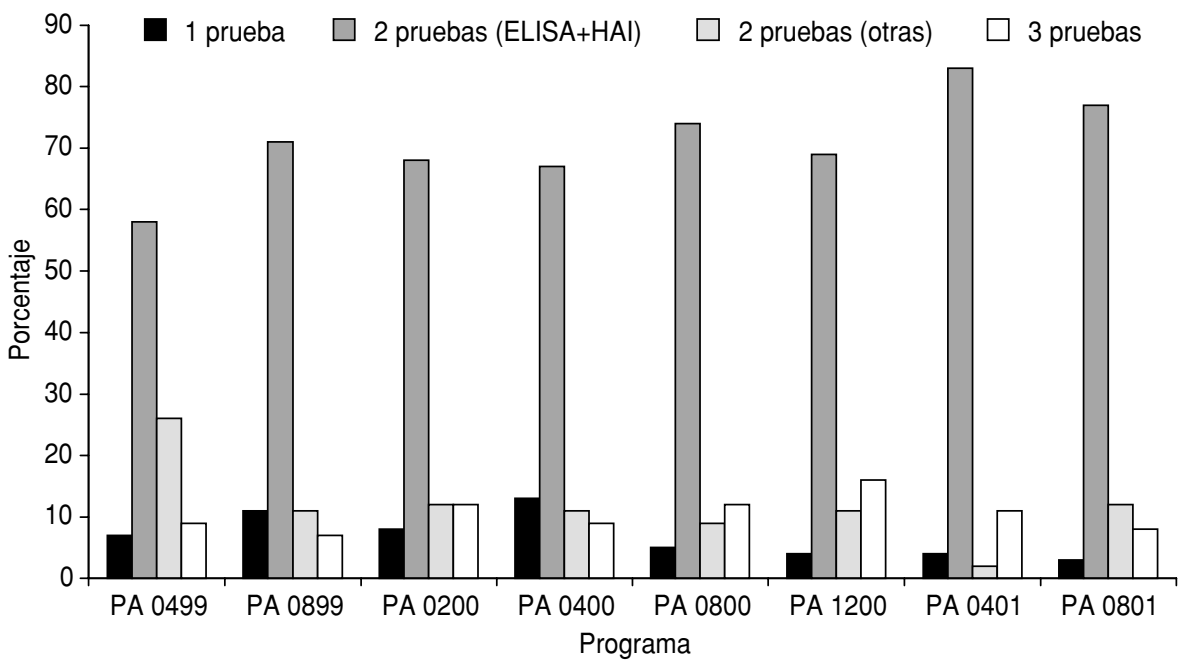

Nota: Por "otras" nos referimos a cualquier asociación diferente de ELISA + hemoaglutinación indirecta (HAI).

FIGURA 3. Evaluación de los laboratorios que participaron en los programas de control de calidad externos de laboratorios de serología, Brasil, 1999-2001

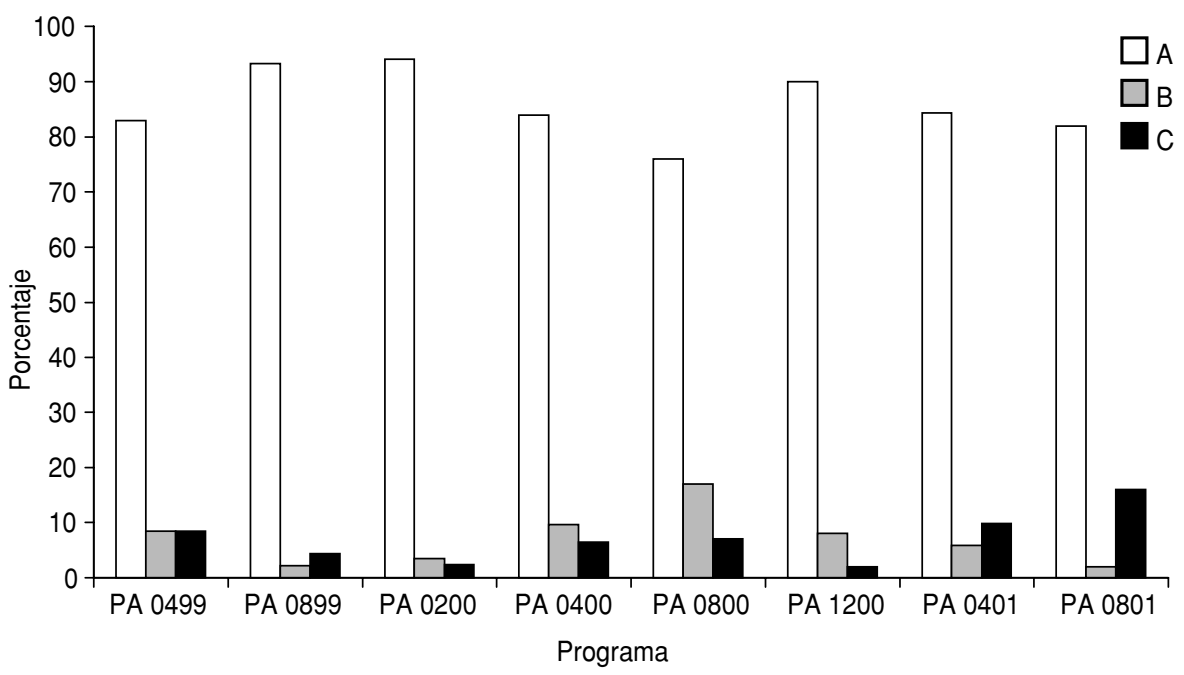

Nota: A: Todos los resultados son correctos; no hay resultados positivos ni negativas falsos. B: Solo se contemplan los resultados positivos falsos. C: Solo se contemplan los resultados negativos falsos.

participantes en el programa y el número de laboratorios en que los resultados negativos falsos hicieron que alguna muestra positiva no fuese detectada. La distribución de los resultados negativos falsos y resultados positivos falsos observados en cada programa puede verse en la figura 4.

No se constató ninguna asociación entre los resultados negativos falsos observados y una muestra o sistema de diagnóstico en particular. La técnica de HAI generó el mayor número de resultados negativos falsos (cuadro 4). La distribución de los resultados negativos falsos por tipo de técnica, en cada programa, se presenta en la figura 5.

Resultados positivos falsos. En 32855 determinaciones realizadas durante los ocho programas en muestras de sueros negativas a anti- $T$. cruzi se obtuvieron $106(0,32 \%)$ resultados positivos falsos. El porcentaje de laboratorios participantes que obtuvo un solo resultado positivo falso por programa estuvo entre el $1 \%$ y el $16 \%$, mientras que entre $0 \%$ y $4 \%$ tuvo más de un resultado positivo falso.

\section{DISCUSIÓN}

Los datos de este trabajo corresponden al desarrollo de ocho PCCES en todo el país, con la participación de alrededor de cien laboratorios por programa, más de $70 \%$ de los cuales se dedican al tamizaje serológico en bancos de sangre. De esta forma se puede considerar que se alcanzó una representatividad adecuada del tamizaje de T. cruzi en Brasil.

Los PCCES sirven para evaluar el desempeño de los laboratorios que efectúan el tamizaje serológico. Se recomienda que los laboratorios participen en esos programas, que en general tienen una periodicidad de 2 a 3 veces al año, para verificar si funcionan adecuadamente sus procedimientos internos de control de calidad y de buenas prácticas de laboratorio.

La herramienta de trabajo fundamental es un panel de sueros enmascarados que se envía a todos los participantes del programa para que los procesen junto con las muestras de donantes de sangre. Es importante que el procesamiento del panel se realice en las mismas condiciones de la rutina diaria de los laboratorios, pues si en el afán por conseguir mejores resultados se procesan de modo diferente - con mayores cuidados que los habitualespueden dejar de detectarse errores que estarán presentes en el trabajo cotidiano del laboratorio. 
CUADRO 3. Distribución de los resultados negativos falsos (RNF) observados en los ocho programas de control de calidad externo de laboratorios de serología. Brasil, 1999-2001

\begin{tabular}{|c|c|c|c|c|}
\hline Programa & LP & $\mathrm{RNF}^{\mathrm{a}}$ & LP con RNFb (\%) & $\begin{array}{l}\text { LP que no detectaron } \\
\text { muestras }{ }^{c}(\%)\end{array}$ \\
\hline PA0499 & 94 & 11 & $8(8,5)$ & $2(2,1)$ \\
\hline PA0899 & 90 & 5 & $4(4,4)$ & $1(1,1)$ \\
\hline PA0200 & 85 & 7 & $2(2,4)$ & $2(2,4)$ \\
\hline PA0400 & 94 & 8 & $6(6,4)$ & $1(1,1)$ \\
\hline PA0800 & 100 & 10 & 7 (7) & 0 \\
\hline PA1200 & 103 & 3 & $2(1,9)$ & $1(1)$ \\
\hline PA0401 & 102 & 18 & $10(9,8)$ & $3(2,9)$ \\
\hline PA0801 & 116 & 23 & $19(16,4)$ & $4(3,4)$ \\
\hline Total & 784 & 85 & $58(7,4)$ & $14(1,8)$ \\
\hline
\end{tabular}

Nota: LP: laboratorios participantes que devolvieron los resultados.

a Número total de RNF a anti-T. cruzi por programa.

b Número total de laboratorios participantes, por programa, que tuvieron RNF a anti-T. cruzi.

c Número de laboratorios participantes, por programa, que tuvieron RNF a anti-T. cruzi y no consiguieron identificar ninguna muestra positiva con las pruebas utilizadas.

FIGURA 4. Distribución de los resultados negativos falsos (RNF) y resultados positivos falsos (RPF) observados en cada uno de los programas de control de calidad externos de laboratorios de serología, Brasil, 1999-2001

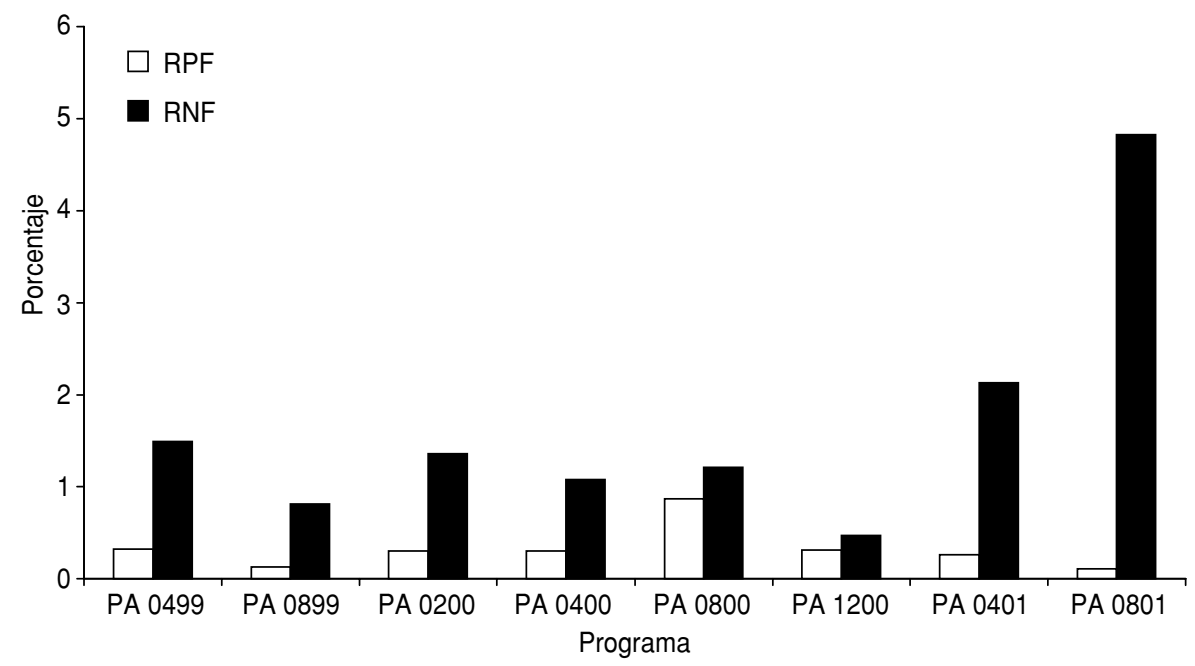

El tamizaje serológico para evitar la transmisión de T. cruzi mediante transfusiones sanguíneas es un tema de importancia fundamental en toda América Latina, desde la frontera de México con los EE.UU. hasta el sur de Argentina y Chile. Para que ese tamizaje sea realmente eficaz es importante que sea obligatorio someter a tamizaje todas las donaciones de sangre con sistemas diagnósticos de buena calidad (previamente evaluados) y que haya programas de evaluación externa para comprobar la eficacia del tamizaje.

En Brasil, es obligatorio desde 1989 realizar dos pruebas de principios distintos para el tamizaje de anti-T. cruzi en donantes de sangre (4). Las pruebas serológicas utilizadas en este tamizaje continúan siendo prácticamente las mismas de los últimos 12 años: HAI, IFI y ELISA.

El comportamiento de las pruebas de HAI, IFI y ELISA frente a paneles de sueros positivos y negativos ha sido bien estudiado, y se conocen los índices de sensibilidad y especificidad de cada una de ellas. Para eso se utilizan muestras de suero provenientes de personas con resultados serológicos positivos y de personas con resultados negativos sin historia de exposición al vector, o sea, un muestreo limitado.

En los bancos de sangre, cuando se utiliza una combinación de dos o más pruebas para el tamizaje se observan innumerables resultados discrepantes entre ellas (5). Como todavía no existe un consenso respecto a las pruebas confirmatorias para el diagnóstico seroló-

CUADRO 4. Resultados negativos falsos observados en el tamizaje de anti-Trypanosoma cruzi, según las técnicas utilizadas en los programas de control de calidad externos para laboratorios de serología. Brasil, 1999-2001

\begin{tabular}{|c|c|c|c|c|c|c|c|c|}
\hline \multirow[b]{3}{*}{ Técnica } & \multicolumn{8}{|c|}{ Programa } \\
\hline & PA0499 & PA0899 & PA0200 & PA0400 & PA0800 & PA1200 & PA0401 & PA0801 \\
\hline & No./total (\%) & No./total (\%) & No./total (\%) & No./total (\%) & No./total (\%) & No./total (\%) & No./total (\%) & No./total (\%) \\
\hline ELISA & $1 / 344(0,3)$ & $1 / 290(0,3)$ & $1 / 238(0,4)$ & $2 / 356(0,6)$ & $1 / 368(0,3)$ & $1 / 285(0,3)$ & $6 / 404(1,5)$ & $6 / 228(2,6)$ \\
\hline IFI & $2 / 128(1,6)$ & $1 / 54(1,8)$ & $1 / 52(1,9)$ & $0 / 68(0)$ & $3 / 80(3,8)$ & $0 / 72(0)$ & $0 / 44(0)$ & $1 / 44 \quad(2,3)$ \\
\hline $\mathrm{HAl}$ & $8 / 268 \quad(2,9)$ & $3 / 269(1,1)$ & $5 / 224 \quad(2,2)$ & $6 / 312(1,9)$ & $6 / 367(1,7)$ & $2 / 285(0,7)$ & $12 / 396(3,0)$ & $16 / 202(7,9)$ \\
\hline$A G$ & $0 / 8(0)$ & $0 / 3(0)$ & $0 / 3(0)$ & $0 / 4(0)$ & $0 / 4(0)$ & $\ldots$ & $\ldots$ & $0 / 2(0)$ \\
\hline$A L$ & $\cdots$ & $\cdots$ & . & $\cdots$ & $0 / 4(0)$ & $\ldots$ & $\cdots$ & $\cdots$ \\
\hline Total & $11 / 748(1,5)$ & $5 / 616(0,8)$ & $7 / 517 \quad(1,3)$ & $8 / 740(1,1)$ & $10 / 823(1,2)$ & $3 / 642(0,5)$ & $18 / 844(2,1)$ & $23 / 476(4,8)$ \\
\hline
\end{tabular}

Nota: ELISA: ensayo de inmunoadsorción enzimática; IFI: inmunofluorescencia indirecta; HAl: hemoaglutinación indirecta; AG: aglutinación en gel; AL: aglutinación en látex. 
FIGURA 5. Distribución de los resultados negativos falsos obtenidos en los programas de control de calidad externos de laboratorios de serología, según la técnica empleada. Brasil, 1999-2001

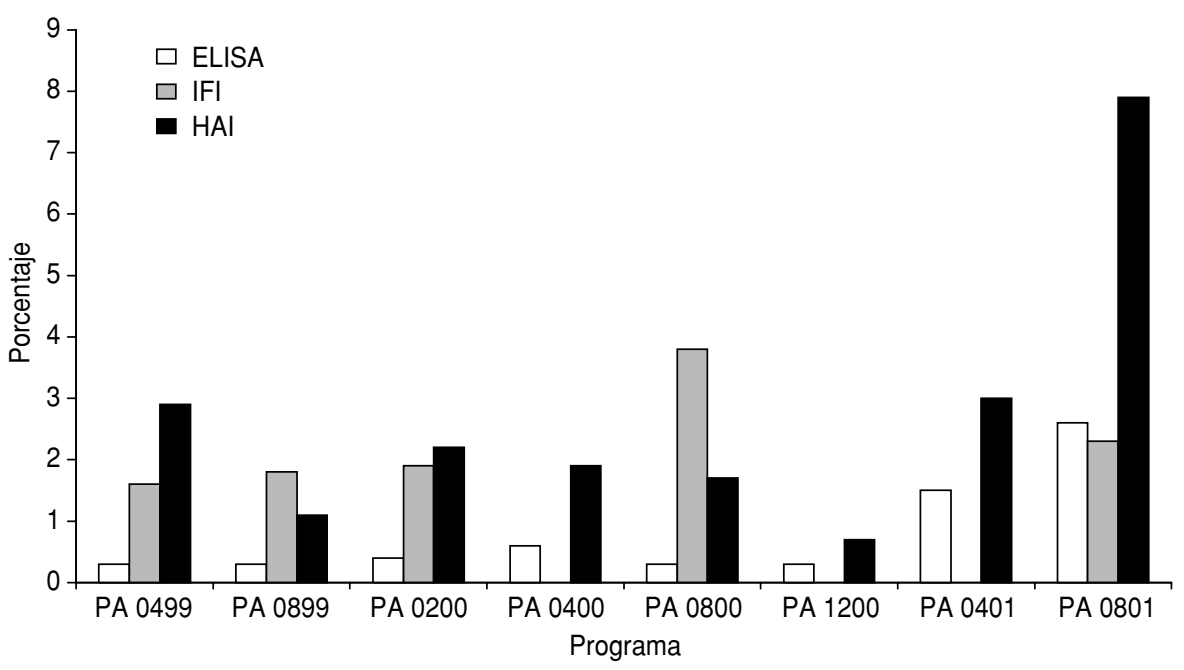

Nota: IFI: inmunofluorescencia indirecta; HAl: hemoaglutinación indirecta.

gico de la infección por T. cruzi, esos resultados discrepantes en el tamizaje constituyen un verdadero problema, por lo que se continúan utilizando dos pruebas diferentes en el tamizaje habitual de donantes de sangre.

Un estudio realizado en Brasil en 1994-1995 para evaluar el tamizaje de la enfermedad de Chagas en 57 bancos de sangre públicos (6) mostró que había una gran mejoría en comparación con estudios anteriores (7). En esa ocasión se observaron $64(3,7 \%)$ resultados negativos falsos en un total de 1725 determinaciones en muestras positivas a anti-T. cruzi. También se pudo verificar que la estrategia de tamizaje utilizada por más del $50 \%$ de los bancos de sangre era la combinación de una prueba de ELISA con una prueba de HAI.

En el presente trabajo se encontraron $85(1,6 \%)$ resultados negativos falsos en un total de 5406 determinaciones realizadas en muestras positivas a antiT. cruzi. De acuerdo con el análisis de regresión efectuado, es posible que la proporción de resultados negativos falsos siga una tendencia general descendente desde los programas de 1994-1995, según las pruebas más utilizadas (ELISA, HAI y IFI). Con res- pecto a los métodos de aglutinación en gel y de aglutinación en látex, es imposible llegar a una conclusión debido al pequeño número de observaciones (cuadro 4).

Al mismo tiempo se puede observar que la gran mayoría de los laboratorios participantes $(83,6 \%-98 \%)$ no obtuvieron resultados negativos falsos. Por otro lado, no todos los laboratorios que notificaron resultados negativos falsos $(2 \%-16,4 \%)$ se vieron afectados en su tamizaje, pues solamente 14 laboratorios (de 0 a 4 por programa) dejaron pasar alguna muestra positiva por no detectarla con ninguna de las pruebas de tamizaje utilizadas (cuadro 3), y apenas un porcentaje muy pequeño de los laboratorios participantes $(0 \%-3,4 \%$ por programa) dejó de detectar muestras positivas a anti-T. cruzi. Al mismo tiempo, el análisis estadístico mostró que esos porcentajes no difieren entre sí de un modo significativo a lo largo de los ocho programas desarrollados.

La prueba ELISA continúa siendo la más utilizada $(92 \%-98 \%)$ y la combinación de una prueba ELISA con HAI aún es la estrategia más usada por los bancos de sangre $(58 \%-83 \%)$ (figura 2).
Es posible que exista una tendencia descendente de los resultados negativos falsos en el decorrer de los PCCES. Sin embargo, llama la atención el hecho de que la gran mayoría de los bancos de sangre continúan usando pruebas de HAI para el tamizaje (cuadro 4, figura 2) a pesar de que la mayoría de los resultados negativos falsos observados ocurrieron con esta técnica, al igual que en los programas desarrollados en Brasil entre 1994 y 1995 (6).

En 1997 se publicó un estudio multicéntrico para evaluar las pruebas de HAI comercializadas en Brasil. El estudio demostró que muchas de las pruebas evaluadas no eran adecuadas debido a su baja sensibilidad (8). También llamó la atención que la reproducibilidad de esas pruebas no era constante, y los resultados finales tuvieron que ser decididos por consenso entre cinco laboratorios de referencia que participaron en el programa.

Tomando en cuenta que la calidad de las pruebas de HAI debe haber mejorado desde entonces y que en Brasil desde hace dos años aproximadamente es obligatorio hacer una evaluación previa para obtener la licencia de comercialización de los sistemas de diagnóstico, la falta de reproducibilidad de esas pruebas, asociada al tipo de procedimiento manual y a la lectura visual, podría ser la causa del alto número de resultados negativos falsos.

Tomando en consideración los datos obtenidos en este trabajo y en investigaciones anteriores $(6,8)$, sería aconsejable dar preferencia a las pruebas antiT. cruzi del tipo ELISA para el tamizaje de la infección crónica por T. cruzi en laboratorios de serología de bancos de sangre. Actualmente se comercializan pruebas de ELISA no solo con fracciones antigénicas provenientes de la lisis del parásito, sino también con fracciones antigénicas recombinantes y péptidos sintéticos. Una forma de cumplir con la norma establecida en Brasil — que determina que las dos pruebas utilizadas en el tamizaje se basen en principios diferentes- es utilizar pruebas con fracciones antigénicas distintas cada vez. Por supuesto, sería fundamental realizar estudios 
para la comparación de las diferentes pruebas de ELISA, y esos estudios deberían ser realizados con muestras provenientes del tamizaje serológico diario de donantes de sangre.

Aunque todavía no existe un consenso con respecto a las pruebas que podrían ser utilizadas para confirmar la infección por T. cruzi, algunos métodos de inmunoblot descritos recientemente $(9,10)$ podrían ayudar a elucidar algunos casos de interpretación dudosa por los métodos tradicionales (5).

El criterio de evaluación utilizado en estos PCCES tomó en consideración que la presencia de resultados negativos falsos es mucho más grave que la de resultados positivos falsos. Como en el tamizaje serológico de donantes bridge, MA: Cytel Software Corporation; 1998.

2. Stata Corp. Stata Statistical Software: Release 7.0. College Station, TX: Stata Corporation; 2002.

3. Royston P. PTREND: Stata module for trend analysis for proportions. Boston: Boston College Department of Economics; 2002. http:// fmwww.bc.eduRePec-bocode-p

4. Ministério da Saúde, Brasil. Normas técnicas para coleta, processamento e transfusão de sangue, componentes e derivados. Diário Oficial da União; 19 de novembro 1993, portaria No. 1367.

5. Salles N, Sabino EC, Cliquet MG, Eluf-Neto J, Mayer A, Almeida-Neto C, et al. Risk exposure for Chaga's disease among seroreactive Brazilian blood donors. Transfusion 1996;36: 969-973. de sangre se deben utilizar pruebas con la máxima sensibilidad que permita conservar al receptor, con frecuencia la especificidad no es la ideal y aparecen algunos resultados positivos falsos.

La presencia de resultados positivos falsos constituye un problema importante por el hecho de que se le comunica al donante un resultado erróneo y también porque se pierden unidades de sangre. Sin embargo, aunque deben evitarse, no comprometen la calidad de los hemoderivados liberados para transfusiones. En cambio, la presencia de resultados negativos falsos significa un riesgo real de transmitir la enfermedad.

En el presente estudio, más de $80 \%$ de los laboratorios que participaron en

\section{REFERENCIAS}

6. Sáez-Alquézar A, Otani MM, Sabino EC, Ribeiro-dos-Santos G, Salles N, Chamone DF. Evaluation of the performance of Brazilian blood banks in testing for Chaga's Disease. Vox Sang 1998;74:228-231.

7. Andrade ALSS, Martelli CMT, Luquetti AO, Oliveira OS, Silva SA, Zicker F. Serologic screening for Trypanosoma cruzi among blood donors in central Brazil. Bull Pan Am Health Organ 1992;26:157-164.

8. Sáez-Alquézar A, Luquetti AO, BorgesPereira J, Moreira EF, Gadelha MFS. GarciaZapata MT, et al. Estudo multicêntrico: Avaliação do desempenho de conjuntos diagnósticos de hemaglutinação indireta, disponíveis no Brasil, para o diagnóstico sorológico da infecção pelo Tripanosoma cruzi. Revista de Patologia Tropical 1997;26(2):343-374.

9. Umezawa ES, Nascimento MS, Kesper Jr N, Coura JR, Borges-Pereira J, Junqueira ACV, siete de los ocho programas obtuvieron una calificación de A - que corresponde a la ausencia de resultados positivos falsos y resultados negativos falsos- y en el programa restante (PA0800) 76\% obtuvo esta calificación (figura 3).

Agradecimiento. Les agradecemos a todas las instituciones públicas y privadas que participaron en los PCCES, así como a la Sociedad Brasileña de Hematología y Hemoterapia (SBHH), su apoyo y confianza. Le agradecemos también a Isabel Cristina Machado Silva su ayuda en la confección del manuscrito. et al. Immunoblot assay using excretedsecreted antigens of Trypanosoma cruzi in serodiagnosis of congenital, acute, and chronic Chaga's Disease. J Clin Microbiol 1996;34(9): 2143-2147.

10. Sáez-Alquézar A, Sabino EC, Salles N, Chamone DF, Hulstaert F, Pottel $\mathrm{H}$, et al. Serological confirmation of Chaga's disease by a recombinant and peptide antigen line immunoassay: INNO-LIA Chagas. J Clin Microbiol 2000;38(2):851-854.

Manuscrito recibido el 25 de marzo de 2002. Aceptado para publicación, tras revisión, el 2 de enero de 2003. 
ABSTRACT Objectives. In 1995 a series of external quality control programs (EQCPs) was launched for serology laboratories in Brazil, open to both public and private blood-bank laboratories. These "programs" have been held several times per year since 1995. The objective of this paper is to present the results achieved during eight programs conducted between April 1999 and August 2001, in terms of serological screening for Chagas' disease.

Methods. The participants in these eight EQCPs were serology laboratories from public and private institutions. The number of participants in each respective program that sent in its results was 94,90, 85, 94, 100, 103, 102, and 116. At the beginning of each EQCP a blinded panel of sera was sent to each participating institution. Each panel consisted of 24 serum samples with different reactivities for the markers for which the serological screening of blood donors is obligatory in Brazil; among the 24 samples were some negative ones. The participants had 60 days to complete their testing and send in their results for evaluation. Later, each participant received an answer key for the sera panel, to use in self-evaluation.

Results. The three tests that the participants used most frequently were enzyme-linked immunosorbent assay (ELISA), indirect hemagglutination (IHA), and indirect immunofluorescence (IIF). Of these three, the ELISA was used most often, ranging from $92 \%$ to $98 \%$ of the laboratories for the eight programs. The screening strategy that was most often used was a combination of an ELISA test and an IHA test (ranging from $58 \%$ to $83 \%$ of the laboratories for the eight programs). In the various programs, the large majority of participating laboratories (range, $83.6 \%-98.1 \%$ ) obtained accurate results without any false negative results. Of the 5406 tests carried out during the eight programs using positive anti-T. cruzi sera samples, 85 (1.6\%) were reported as negative in 58 laboratories. However, only 14 of those laboratories were unable to identify one or more positive samples after using all their screening tests. There were no significant differences found between the blood banks in the eight programs $(P=0.5936)$. No association was found between false negative results and any particular sample or any specific diagnostic kit. IHA testing had the highest proportion of false negatives, ranging from $0.7 \%$ to $7.9 \%$ for the eight programs. Of 32855 tests performed during the eight programs using negative anti-T. cruzi sera, $106(0.32 \%)$ were reported as false positives. The percentage of participating laboratories that had one false positive result per program varied from $1 \%$ to $16 \%$, and between $0 \%$ and $4 \%$ of the labs per program had more than one false positive result. In comparison to testing done in Brazil in 1994-1995, the proportion of false negative results has declined.

Conclusions. The results of this study point to a decrease over time in the proportion of false negative results. In the 1999-2001 programs, the highest percentage of false negative results was found with IHA testing. Therefore, it is recommended to give preference to ELISA testing for screening blood donors.

By the ancients man has been called the world in miniature; and certainly this name is well bestowed, because, inasmuch as man is composed of earth, water, air and fire, his body resembles that of the earth; as man has in him bones the supports and framework of his flesh, the world has its rocks the supports of the earth; as man has in him a pool of blood in which the lungs rise and fall in breathing, so the body of the earth has its ocean tide which likewise rises and falls every six hours, as if the world breathed; as in that pool of blood veins have their origin, which ramify all over the human body, so likewise the ocean sea fills the body of the earth with infinite springs of water.

Leonardo da Vinci, The Notebooks 